\title{
FISIOLOGIA PÓS-COLHEITA DO CAMU-CAMU TRATADO COM DIFERENTES FONTES E DOSES DE RADIAÇÃO
}

\author{
Alex Guimarães Sanches ${ }^{1}$, Amanda Germano Silveira ${ }^{2}$, Maryelle Barros da Silva ${ }^{1}$, Elaine Gleice Silva Moreira ${ }^{1}$ \\ ${ }^{1}$ Universidade Federal do Pará - UFPA. ${ }^{2}$ Universidade Federal do Ceará - UFC. E-mail: \\ alexsanches.eng@gmail.com
}

\section{RESUMO}

Por se tratar de um fruto climatérico, o camu-camu (Myrciaria dubia (H.B.K.) McVaugh) apresenta uma curta vida útil pós-colheita, principalmente quando armazenado em temperatura ambiente (>25 ㄷ), observando excessiva perda de umidade e aumento na atividade respiratória e na produção de etileno que aceleram a senescência. Assim, o presente trabalho tem por objetivo avaliar o efeito de diferentes doses de radiação gama e ultravioleta $\mathrm{C}$ sobre a fisiologia e a qualidade pós-colheita do camu-camu. Os frutos foram tratados com radiação gama (1,0 e 2,0 KGy) e radiação ultravioleta $C(1,0$ e 2,0 kJ / m²), em seguida, os mesmos foram armazenados sob refrigeração por 21 dias a $10^{\circ} \mathrm{C}$ e avaliados a cada três dias sobre a: perda de massa fresca, firmeza, sólidos solúveis, acidez titulável, $\mathrm{pH}$, vitamina $\mathrm{C}$ e antocianinas totais. $\mathrm{O}$ tratamento pós-colheita do camu-camu com radiação gama e ultravioleta C (UV-C), especialmente nas doses de $1,0 \mathrm{kGy}$ e $1,0 \mathrm{~kJ} / \mathrm{m}^{2}$, respectivamente é eficiente em preservar a qualidade dos frutos por até 21 dias em ambiente refrigerado e, a exposição às doses de $1,0 \mathrm{kGy}$ e $2,0 \mathrm{~kJ} / \mathrm{m}^{2}$ estimula a síntese de antocianinas durante o armazenamento dos frutos.

Palavras-chave: Myrciaria dubia (H.B.K.) McVaugh; análises físico-químicas;estresse abiótico.

\section{POST-HARVEST PHYSIOLOGY OF CAMU-CAMU TREATED WITH DIFFERENT SOURCES AND RADIATION DOSES}

\begin{abstract}
Because it is a climacteric fruit, camu-camu (Myrciaria dubia (HBK) McVaugh) has a short post-harvest shelf life, especially when stored at room temperature $(>25 \circ \mathrm{C})$, observing excessive moisture loss and increased activity respiratory and ethylene production that accelerate senescence. Thus, the present work aims to evaluate the effect of different doses of gamma and ultraviolet $C$ radiation on the physiology and postharvest quality of camu-camu. The fruits were treated with gamma radiation (1.0 and $2.0 \mathrm{KGy}$ ) and ultraviolet radiation $\mathrm{C}(1.0$ and $2.0 \mathrm{~kJ} / \mathrm{m} 2)$, then they were stored under refrigeration for 21 days at $10^{\circ} \mathrm{C}$ and evaluated every three days on: fresh weight loss, firmness, soluble solids, titratable acidity, $\mathrm{pH}$, vitamin $\mathrm{C}$ and total anthocyanins. The post-harvest treatment of camu-camu with gamma and ultraviolet $\mathrm{C}$ (UV-C) radiation, especially at $1.0 \mathrm{kGy}$ and $1.0 \mathrm{~kJ} / \mathrm{m} 2$, respectively, is efficient in preserving fruit quality for up to 21 days in a refrigerated environment, and exposure to the $1.0 \mathrm{kGy}$ and $2.0 \mathrm{~kJ} / \mathrm{m} 2$ doses stimulates anthocyanin synthesis during fruit storage.
\end{abstract}

Keywords: Myrciaria dubia (HBK) McVaugh); physical and chemical analysis; abiotic stress.

\section{INTRODUÇÃO}

Fruto de planta nativa da Amazônia brasileira e peruana, o camu-camu, pertencente à família Myrtaceae, é cultivado, principalmente, perto de rios, córregos ou regiões inundadas permanentemente, onde $\mathrm{o}$ caule permanece submerso (INOUE et al., 2008).Entretanto, estudos têm sido realizados, objetivando a expansão das áreas de cultivo e produção do fruto, visto que o camu-camu apresenta crescente importância comercial (ZANATTA; MERCADANTE, 2007; GRIGIO et al., 2015).

O grande potencial econômico do camucamu é atribuído, principalmente, ao seu valor nutricional, sendo rico em vitamina $\mathrm{C}$ com valores superiores a $2.400 \mathrm{mg} .100 \mathrm{~g}^{-1} \mathrm{em}$ frutos maduros 
(ABANTO-RODRIGUEZ et al., 2016) e em compostos fenólicos como flavonóides, e antocianinas (MYODA et al., 2010; IMÁN et al., 2011). Esses compostos possuem propriedades antioxidantes e propriedades antiinflamatórias, com potencial para combater doenças crônicas induzidas pelo estresse, quando os frutos são consumidos como parte da dieta (FUJITA et al., 2013).

No entanto, o camu-camu por se tratar de um fruto climatérico, apresenta uma rápida deterioração após a colheita atribuída à alta taxa respiratória, o aumento na produção de etileno (CARRILLO et al., 2011; PINTO et al., 2013) e a perda de água reduzindo o período de conservação (YUYAMA et al., 2010). Assim, o uso de uma tecnologia adequada para melhorar sua conservação através da síntese de compostos com propriedades nutracêuticos pode potencializar a comercialização do fruto in natura.

A radiação é uma técnica que tem sido usada em vários países com benefícios na conservação de frutas e vegetais (STEFANOVA et al., 2010). A exposição a baixas doses de radiação pode retardar o amadurecimento e a senescência das frutas e vegetais, aumentando assim a vida útil (CHEN et al., 2012; KHADEMI et al., 2013) e, consequentemente, as possibilidades de comercialização e maximização dos lucros tanto dos produtores como dos comerciantes (FRANÇOSO et al., 2008).

A radiação gama mostra-se bastante eficiente no prolongamento da vida comercial de frutos (CAMPOS et al., 2011). Atrasando processos de amadurecimento e senescência, reduzindo a decomposição sem causar alterações significativas na aparência, sabor e qualidade nutricional (TEZOTTO-ULIANA et al., 2015).A radiação ultravioleta $C$ (UV-C) é um processo seguro e comprovado em muitas aplicações póscolheita. Devido à dose aplicada aos alimentos, observa-se uma melhora na qualidade microbiológica do produto, resultando em perdas de armazenamento reduzidas e vida útil prolongada (ALAM KHAN; ABRAHEM, 2010).

Nesse contexto, o presente trabalho objetiva avaliar os efeitos de diferentes doses de radiação gama e ultravioleta C (UV-C) sobre a fisiologia e a qualidade pós-colheita do camucamu durante $o$ armazenamento refrigerado.

\section{MATERIAIS E MÉTODOS}

Os frutos de camu-camu foram colhidos em um pomar localizado no município de Altamira, Pará,no estádio de maturidade fisiológica utilizando como base para a coleta a coloração dos mesmos(roxo). Os frutos foram transportadosem caixas térmicas para 0 Laboratório de Ecofisiologia do Centro de Estudos Ambientais, localizado na cidade de Altamira, Pará onde foram selecionados quanto à ausência de defeitos fisiológicos e danos mecânicos.

No laboratório, os frutos foram sanitizados por imersão em soluçãode hipoclorito de sódio a $150 \mathrm{mg}^{-1} \mathrm{~L}^{-1}$ durante 5 minutos e, posteriormente secos à temperatura ambiente (25 ㅇ) por 2 horas. Em seguida estes foram divididos em cinco lotes e submetidos airradiação gama e ultravioleta C (UV-C), além do lote que não foi irradiado representado pelo tratamento controle.

Para o tratamento com raios gama os frutos foram irradiados nas doses de 1,0 e 2,0 KGy utilizando um irradiador do tipo GammaCell 220 (Atomica energy of Canada Ltd, Canadá) com fonte de cobalto ${ }^{60}$, sob uma taxa de dose de $0,350 \mathrm{kGy} / \mathrm{h}$ controlada com auxílio de um radiômetro.

Para o tratamento com radiação UV-C os frutos foram dispostos sem sobreposição sobre uma bandeja de vidro translúcida em uma caixa de aço inoxidável e, irradiadas nas doses de 1,0 e $2,0 \mathrm{~kJ} / \mathrm{m}^{2}$ com o auxílio de três lâmpadas de mercúrio de baixa pressão localizadas a uma distância de $30 \mathrm{~cm}$ acima e abaixo da superfície dos frutos. As lâmpadas UV-C foram aquecidas por 30 minutos antes da irradiação para garantir resultados confiáveis. A intensidade de radiação UV-C $(254 \mathrm{~nm})$ foi monitorada usando um radiômetro (Lutron Electronic Co.,Ltd., Taiwan).

Imediatamente após a exposição as fontes irradiantes, os frutos foram dispostos em bandejas de poliestireno expandido (EPS) revestidos com filme plástico de PVC 14 micras e armazenados sob refrigeração $\left(10 \pm 2{ }^{\circ} \mathrm{C}\right) \mathrm{com}$ umidade relativa de $85 \pm 5 \%$, por um período de 21 dias.

Adotou-se um delineamento experimental inteiramente casualizado (DIC), sob esquema fatorial de $5 \times 8$, isto é, com cinco tratamentos (controle; 1,0 e 2,0 KGy de radiação gama; 1,0 e $2,0 \mathrm{~kJ} / \mathrm{m}^{2}$ de radiação ultravioleta $\mathrm{C}$ ) e oito tempos de avaliação $(0$ 3, 6, 9, 12, 15, 18 e 21 dias), cada tratamento foi constituído por 
cinco repetições e a parcela experimental composta por 10 frutos.

Os frutos de cada tratamento foram avaliados a cada três dias quanto: perda de massa fresca determinada pela diferença entre o peso inicial das bandejas contendo 10 frutos e o peso obtido em cada intervalo do tempo de armazenamento sendo os resultados expressos em percentagem (\%).

A firmeza dos frutos foi determinada utilizando um penetrômetro digital, modelo Sammar 53200 (TR Turoni, Forli, Itália), com ponteira de $6 \mathrm{~mm}$. As medições foram realizadas na região equatorial dos 10 frutos e em ambos os lados. Os resultados foram expressos em Newton (N).

O teor de sólidos solúveis totais (SST) foi determinado por leitura refratométrica direta em graus Brix ( ${ }^{\circ}$ Brix), com refratômetro digital (modelo PR 201, ATAGO, USA) com compensação automática de temperatura em $\stackrel{\circ}{ }$.

A acidez total titulável foi medida seguindo a metodologia recomendada pela AOAC (2012), utilizando $10 \mathrm{~g}$ de polpa homogeneizada e diluída em $90 \mathrm{~mL}$ de água destilada, seguindo-se a titulação em uma bureta digital com solução padrão de $0,1 \mathrm{M}$ de $\mathrm{NaOH}$, e a fenolftaleína $1 \%$ como indicador do ponto de viragem. Os resultados foram expressos em \% ácido cítrico em $100 \mathrm{~g}^{-1}$ de massa fresca (MF).

$\mathrm{O} \mathrm{pH}$ foi obtido por leitura direta da polpa triturada em pHmetro digital (TECNAL, modelo MPA 2010), devidamente calibrado em solução tampão 4,0 e 7,0 conforme o AOAC (2012).

O teor de vitamina C foi determinado pelo método proposto por Chen e Wang (2002) em um espectrofotômetro a $525 \mathrm{~nm}$, sendo os resultados expressos em $\mathrm{mg}$ de ácido ascórbico. $100 \mathrm{~g}^{-1}$ de massa fresca com base em uma curva de calibração.

A quantificação de antocianinas foi realizada de acordo com o método do $\mathrm{pH}$ diferencial adaptado de Teixeira, Stringheta e

Oliveira (2008). A polpa camu-camu homogeneizada foi centrifugada a $8.000 \mathrm{xg}$ a $4{ }^{\circ} \mathrm{C}$ durante 20 minutos. Após centrifugação, o sobrenadante foi removido e colocado em tubos de ensaio. Preparou-se duas soluções, uma com adição de $1,0 \mathrm{~mL}$ de solução tampão $\mathrm{KCl}$ a 0,025 $\mathrm{M} \mathrm{pH} \mathrm{1,0} \mathrm{e} \mathrm{1,5} \mathrm{mL} \mathrm{da} \mathrm{polpa} \mathrm{centrifugada;} \mathrm{e} \mathrm{a}$ outra com $1,0 \mathrm{~mL}$ de tampão acetato de sódio a $0,4 \mathrm{M} \mathrm{pH} 4,5$ e 1,5 mL da polpa centrifugada. As leituras espectrofotométricas foram realizadas a $520 \mathrm{~nm}$ e a $700 \mathrm{~nm}$ em ambas as soluções tampão. Os resultados foram expressos em $\mathrm{mg}$ de cianidina-3-glucósideo $\mathrm{g}^{-1}$ de massa fresca.

Os dados foram submetidos à ANOVA e as comparações múltiplas entre as médias dos parâmetros estudados foram realizadas usando o teste de Tukey no nível de probabilidade de 5\% pela interface do software estatístico Assistat 7.7 beta (SILVA; AZEVEDO, 2016).

\section{RESULTADOS E DISCUSSÃO}

A avaliação da perda de massa fresca indicou que o tratamento controle apresentou as percentagens médias mais altas, diferindo significativamente $(p>0,05)$ dos tratamentos nos quais houve aplicação de radiação. Nos frutos expostos as fontes irradiantes, não foram observadas diferença estatística $(p<0,05)$ entre asdosagens avaliadas ao longo do tempo de armazenamento (Figura 1). 
Figura 1. Perda de massa fresca (\%) em camu-camu tratados com diferentes doses e fontes de radiação e armazenados sob refrigeração $10 \pm 2{ }^{\circ} \mathrm{C}$ e $85 \pm 5 \%$ de U.R por 21 dias. Médias seguidas pelas mesmas letras minúsculas (dias de avaliação) e maiúsculas (tratamentos)não diferem entre si pelo teste de Tukey no nível de probabilidade de $5 \%$.

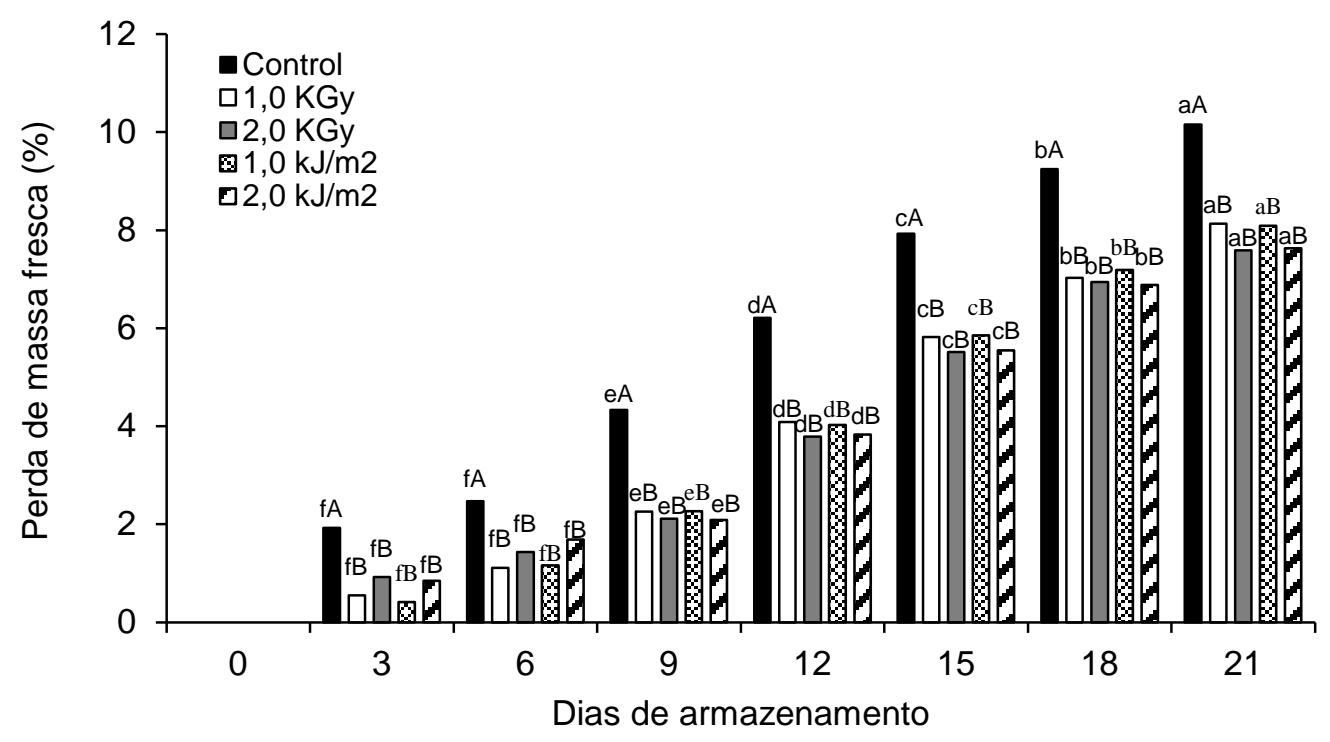

Resultados semelhantes foram obtidos por Guimarães et al. (2013), que durante o armazenamento à 1 oc de framboesas tratadas com radiação gama nas doses de 0,$5 ; 1,0$ e 2,0 kGy apresentaram menor perda de massa fresca $2,25 \%$ ao final de 12 dias quando comparado ao controle,média de $5,10 \%$.

Em abacates tratados com radiação UV-C nas doses de $\left(0,2,0,4\right.$ e $\left.0,6 \mathrm{~kJ} / \mathrm{m}^{2}\right)$, a perda de massa fresca foi menor, média de $(6,4 \%)$ em relação ao controle $(9,1 \%)$ após 12 dias de armazenamento à 10C (DAIUTO et al., 2010).

Sanches et al. (2017) obtiveram uma correlação positiva entre o tempo de armazenamento e a perda de massa fresca em araças (Eugenia stipitata L.). Neste trabalho os frutos expostos por 3 e 6 minutos a fluência de $1.7 \mathrm{~kJ} / \mathrm{m}^{2}$ de radiação UV-C reduziu a perda de massa $(5,5 \%)$ em relação ao controle $(8,8 \%)$ após 12 dias de armazenamento a 25 ㅇ․ .

Em relação ao tempo de armazenamento, observam-se maiores variações, na perda de massa a partir do sexto dia nos frutos do tratamento controle e aos nove dias para aqueles expostos a radiação, independente da fonte (gama e UV-C) utilizada. Ao final de 21 dias, a perda de massa variou entre 7,89 e $8,13 \%$ nos frutos irradiados, não diferindo entre si $(p<0,05)$, enquanto nos frutos do tratamento controle o valor médio excedeu $10 \%$.

A firmeza dos frutos reduziu com o tempo de armazenamento, independente da dose e da fonte irradiante utilizada (gama ou UVC) (Figura 2). 
Figura 2. Firmeza $(\mathrm{N})$ em camu-camu tratado com diferentes doses e fontes de radiação e armazenados sob refrigeração $10 \pm 2{ }^{\circ} \mathrm{C}$ e $85 \pm 5 \%$ de U.R por 21 dias. Médias seguidas pelas mesmas letras minúsculas (dias de avaliação) e maiúsculas (tratamentos)não diferem entre si pelo teste de Tukey no nível de probabilidade de $5 \%$.

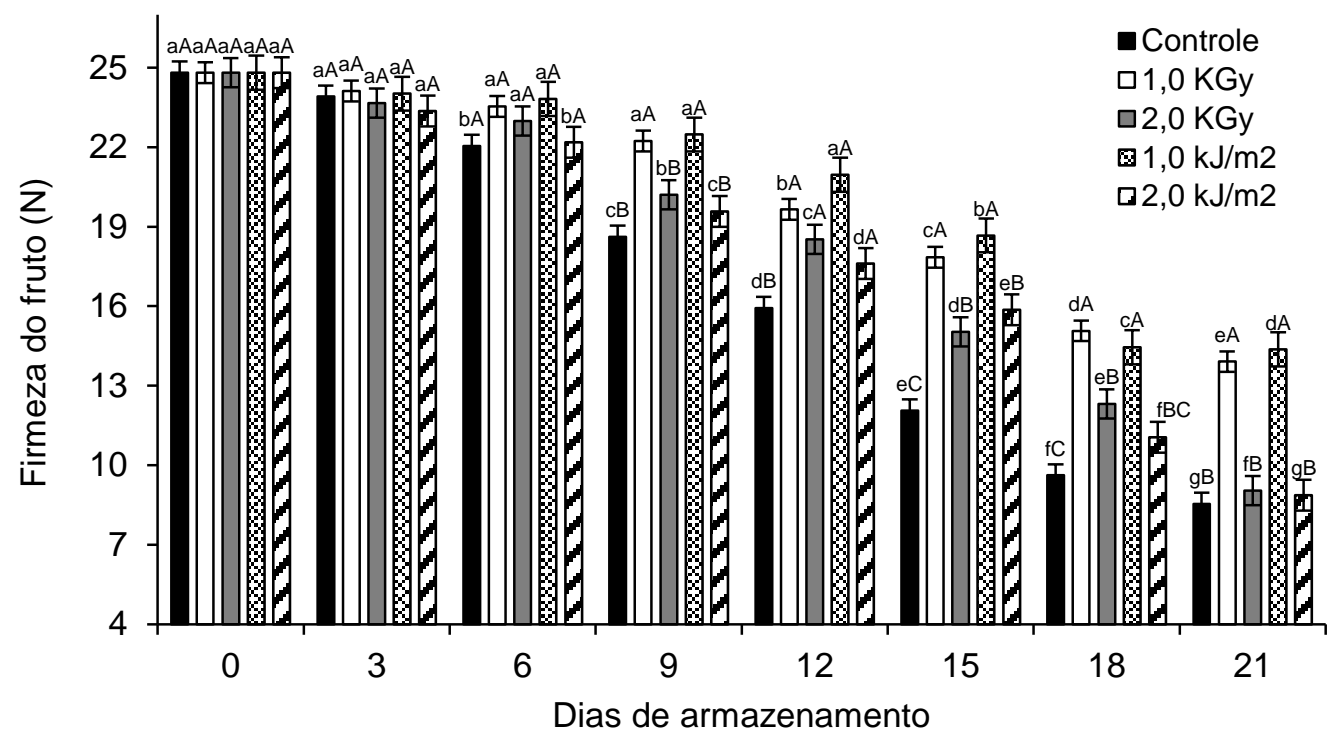

Em geral, entre o tempo zero e o sexto dia de armazenamento não há diferença significativa $(p<0,05)$ entre os tratamentos. No entanto, a partir do nono dia observa-se um decréscimo expressivo nos valores de firmeza, cerca de $80 \%$ aos 21 dias de armazenamento.

Nos frutos irradiados, houve diferença significativa $(p>0,05)$ a partir do $12 \circ$ dia de armazenamento. Em síntese, os frutos irradiados com 2,0 kGy e $2,0 \mathrm{~kJ} / \mathrm{m}^{2}$ apresentaram reduções expressivas na firmeza $(78,7 \%)$ após 21 dias, não diferindo em relação ao controle. Considerando o mesmo intervalo de tempo ( 0 a 21 dias) a redução observada nos frutos irradiados com 1,0 $\mathrm{kGy}$ e $1,0 \mathrm{~kJ} / \mathrm{m}^{2}$ foi de apenas $53,05 \%$.

$\mathrm{Na}$ pós-colheita a radiação pode promover efeitos indesejáveis, tais como: escurecimento dos tecidos, perda de firmeza, sabor e aroma dos produtos, além de depressões superficiais e amadurecimento desregulado (FAN et al., 2012).

Considerando que cada produto possui uma resposta específica aos tratamentos com radiação, é fundamental estabelecer a dose ideal para cada espécie e cultivar, assim como a dosagem de $1,0 \mathrm{kGy}$ e $1,0 \mathrm{~kJ} / \mathrm{m}^{2}$ observada nesta pesquisa, considerada eficaz em retardar a degradação da parede celular dos camu-camu ao longo de 21 dias de armazenamento a $10 \stackrel{\circ}{ } \mathrm{C}$.

Em uvas "Itália" (CAMPOS; VIEITES, 2010) e mangas (YADAV et al., 2015) tratadas com radiação gama, as doses de 0,2 e 0,4 kGy mostraram-se eficientes em preservar a firmeza dos frutos ao longo de 28 e 36 dias de armazenamento à 5 o $C$, respectivamente. Em morangos (POMBO et al., 2009), cogumelos shitake (JIANG et al., 2010) e tangerinas (SANCHES et al., 2017) foi demonstrado que a exposição a radiação UV-C manteve a firmeza maior que o controle pela redução nas atividades de enzimas que degradam a parede celular.

Houve variações no conteúdo de sólidos solúveis (SS) entre os tratamentos durante o período de armazenamento dos frutos (Figura 3). 
Figura 3. Sólidos solúveis totais ( $\left.{ }^{\circ} B r i x\right)$ em camu-camu tratados com diferentes doses e fontes de radiação e armazenados sob refrigeração $10 \pm 2{ }^{\circ} \mathrm{C}$ e $85 \pm 5 \%$ U.R por 21 dias. Médias seguidas pelas mesmas letras minúsculas (dias de avaliação) e maiúsculas (tratamentos) não diferem entre si pelo teste de Tukey no nível de probabilidade de $5 \%$.

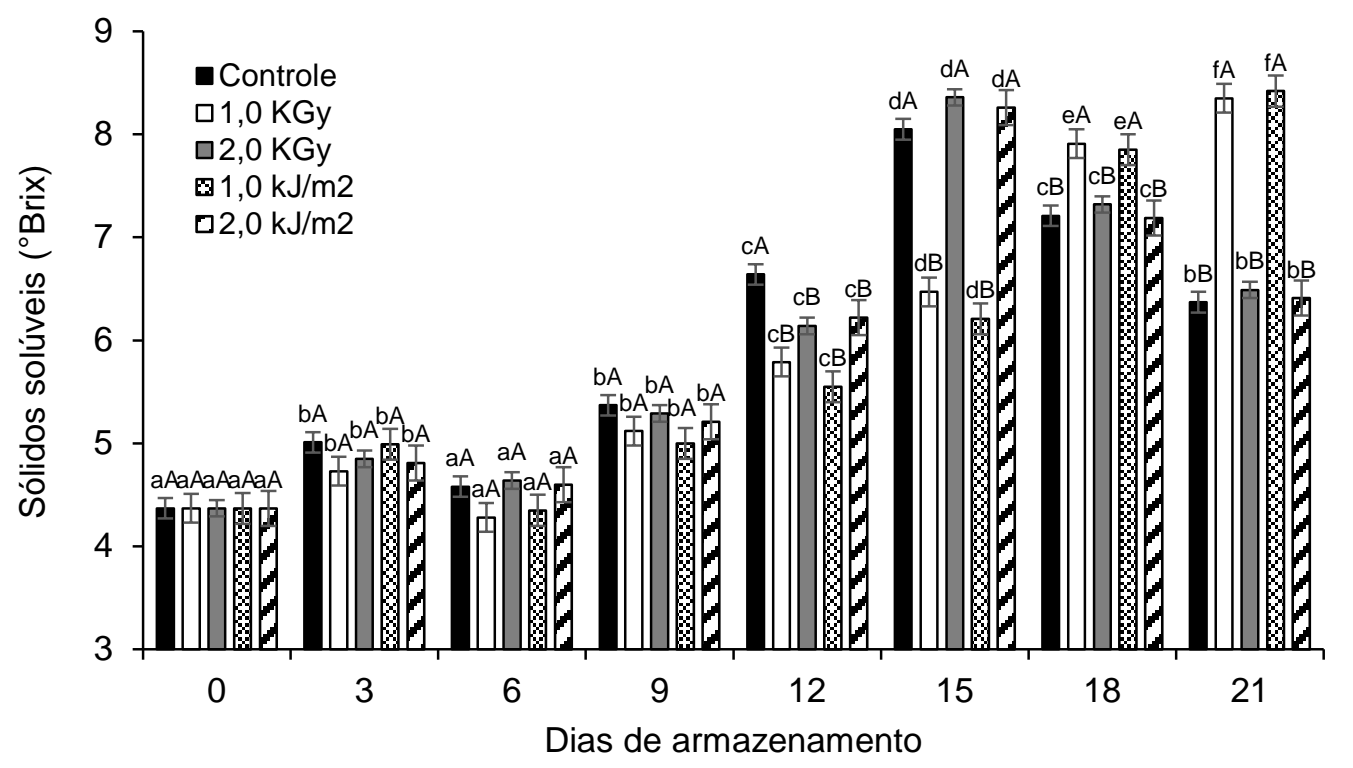

Os valores médios de SS variaram de 4,17 a 8,42 으ix entre o dia zero e o 21 o dia de armazenamento, respectivamente. Pinto et al. (2013) avaliando camu-camu colhidos no estádio de maturação 3 e armazenados a 22 oC obtiveram variações de SS entre 6,09 e 8,85 @Brix ao longo de 12 dias.

Em relação ao tempo de armazenamento, não se observa diferença significativa $(p<0,05)$ entre os tratamentos por até nove dias. Até $15^{\circ}$ dia o conteúdo de SS aumenta nos frutos controle e quando tratados na dose de 2,0 kGy e $2,0 \mathrm{~kJ} / \mathrm{m}^{2}$ atingindo valores máximos de 8,31 ; 8,36 e 8,34 oBrix, respectivamente. Entre o 15 e o 21 ㅇ dia observa-se redução nos valores de SS sugerindo que os açucares passaram a ser utilizados no metabolismo respiratório, sendo, portanto, um indicativo de perda de qualidade "sabor" dos frutos.

Por outro lado, a dosagem de 1,0 kGy e $1,0 \mathrm{~kJ} / \mathrm{m}^{2}$ manteve linear o acréscimo de SS durante todo o período de armazenamento dos frutos observando valor máximo de 8,39 e 8,42 oBrix no 21임.

Considerando o armazenamento póscolheita o aumento no conteúdo de SS em alguns pontos ocorre devido à transformação do amido em açúcares, por causa das condições atmosféricas dentro da embalagem, levando ao amadurecimento, ou da perda de umidade levando ao acúmulo de açucares na parede celular. Entretanto, a diminuição dos SS deve-se a utilização dos açúcares durante a respiração, sendo uma característica de senescência (CHITARRA; CHITARRA, 2005).

Os valores médios de acidez titulável (AT) foram marcados por estabilidade inicial seguido de redução com o avanço do tempo de armazenamento (Figura 4). 
Figura 4. Acidez titulável (\% de ácido cítrico. $100 \mathrm{~g}^{-1} \mathrm{MF}$ ) em camu-camu tratados com diferentes doses e fontes de radiação e armazenados sob refrigeração $10 \pm 2{ }^{\circ} \mathrm{C}$ e $85 \pm 5 \%$ de U.R por 21 dias. Médias seguidas pelas mesmas letras minúsculas (dias de avaliação) e maiúsculas (tratamentos) não diferem entre si pelo teste de Tukey no nível de probabilidade de $5 \%$.

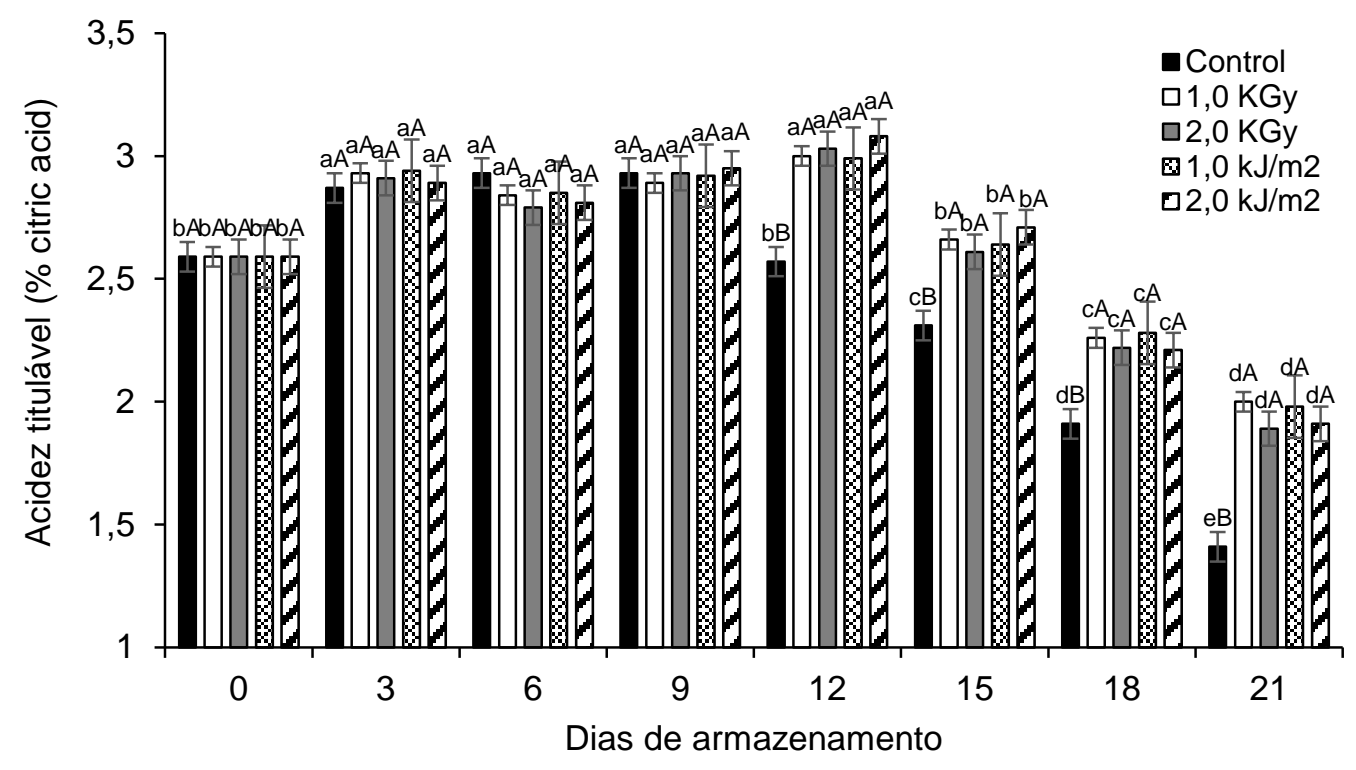

De maneira geral, os valores de AT foram relativamente elevados com variações entre o dia zero e o 21 을 dia de armazenamento de 2,59 a $1,42 \%$ de ácido cítrico.100 $\mathrm{g}^{-1} \mathrm{MF}$, respectivamente.

Avaliando camu-camu colhidos na maturidade fisiológica (estádio 4), Pinto et al. (2013) encontraram valores de $2,65 \%$ de ácido cítrico.100 g $\mathrm{g}^{-1} \mathrm{MF}$. Já Oliveira et al. (2014) verificaram valores superiores a 3,40\% de ácido cítrico.100 $\mathrm{g}^{-1} \mathrm{MF}$ na polpa de camu-camu maduros. Por sua vez, Grigio et al. (2017) encontraram valores de $4,86 \%$ de ácido cítrico.100 $\mathrm{g}^{-1} \mathrm{MF}$ em frutos colhidos na maturidade fisiológica no estado de Roraima. Tal fato confirma a característica ácidae a grande variabilidade genética existente nos constituintes voláteis do fruto.

Nota-se estabilidade nos valores médios de AT até o nono dia de armazenamento, sem diferença significativa entre os tratamentos $(p<0,05)$. Entre o $12^{\circ}$ e o 21 ㅇ dia é marcado por redução da acidez na polpa dos frutos, especialmente naqueles do tratamento controle cuja média foi de $1,42 \%$ de ácido cítrico. $100 \mathrm{~g}$ ${ }^{1} \mathrm{MF}$ ao final de 21 dias. Para o mesmo dia os valores de AT nos frutos irradiados variaram de 1,89 a 2,05\% de ácido cítrico.100 $\mathrm{g}^{-1} \mathrm{MF}$, não diferindo entre si.

O comportamento climatérico através da síntese e acúmulo de ácido orgânicos na polpa dos frutos pode ter contribuído para essa estabilidade inicial observada nos valores de AT. Segundo Chitarra e Chitarra (2005), a redução da AT durante o amadurecimento de frutos é um processo natural, pois os ácidos orgânicos são utilizados como substrato energético (ATP) no metabolismo respiratório. Dessa forma, a exposição dos frutos as fontes irradiantes promoveu alterações fisiológicas como o atraso da maturação dado o menor consumo dos ácidos orgânicos durante $\mathrm{o}$ armazenamento.

Os valores de $\mathrm{pH}$ apresentaram variações com o tempo de armazenamento dos frutos, no entanto, não foi observada interação significativa $(p<0,05)$ entre os tratamentos (Figura 5$)$. 
Figura 5. Valores de $\mathrm{pH}(\mathrm{H}+)$ em camu-camu tratados com diferentes doses e fontes de radiação e armazenados sob refrigeração $10 \pm 2{ }^{\circ} \mathrm{C}$ e $85 \pm 5 \%$ de U.R durante 21 dias. Médias seguidas pelas mesmas letras minúsculas (dias de avaliação) e maiúsculas (tratamentos), não diferem entre si pelo teste de Tukey no nível de probabilidade de $5 \%$.

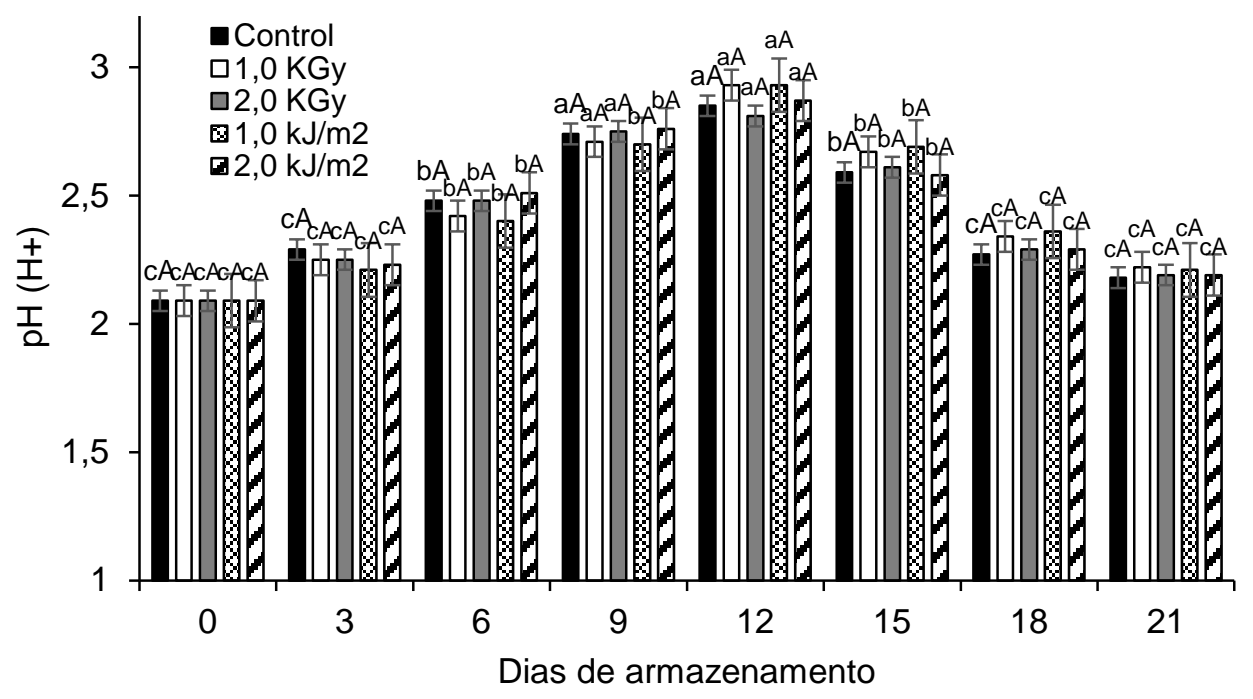

$\mathrm{O} \mathrm{pH}$ nos frutos do camu-camu são abacates, tomates e pitaias (DAIUTO et al., 2010; considerados baixos, uma vez que os valores médios oscilaram entre o dia zero e o 21 dia entre 2,09 a 2,92, respectivamente. Esse baixo valor de $\mathrm{pH}$ está relacionado com o caráter ácido do próprio fruto, que tanto limita o crescimento de microorganismos quanto favorece maior estabilidade do ácido ascórbico em pH ácido, justificando assim, as grandes concentrações desse composto no camu-camu.

A aplicação de diferentes doses de radiação gama em framboesas (GUIMARÃES et al., 2013), limas ácidas (SILVA et al., 2015) e PATARO et al., 2015; MINITKEATKAl; KULTHIP, 2016) tratados com radiação UV-C, não foi observado efeito significativo das doses e fontes irradiantes sobre $\mathrm{o} \mathrm{pH}$ dos frutos, havendo, contudo, redução nos valores destes com o tempo de armazenamento, similar ao verificado neste trabalho.

O conteúdo de vitamina $\mathrm{C}$ reduziu significativamentedurante $\mathrm{o}$ tempo de armazenamento, independentemente da dose e fonte irradiante utilizada (Figura 6).

Figura 6. Teor de vitamina C ( $\mathrm{mg}$ de AsA.100 $\mathrm{g}^{-1} \mathrm{MF}$ ) em camu-camu tratados com diferentes doses e fontes de radiação e armazenados sob refrigeração $10 \pm 2{ }^{\circ} \mathrm{C}$ e $85 \pm 5 \%$ U.R por 21 dias. Médias seguidas pelas mesmas letras minúsculas (dias de avaliação) e maiúsculas (tratamentos), não diferem entre si pelo teste de Tukey no nível de probabilidade de $5 \%$.

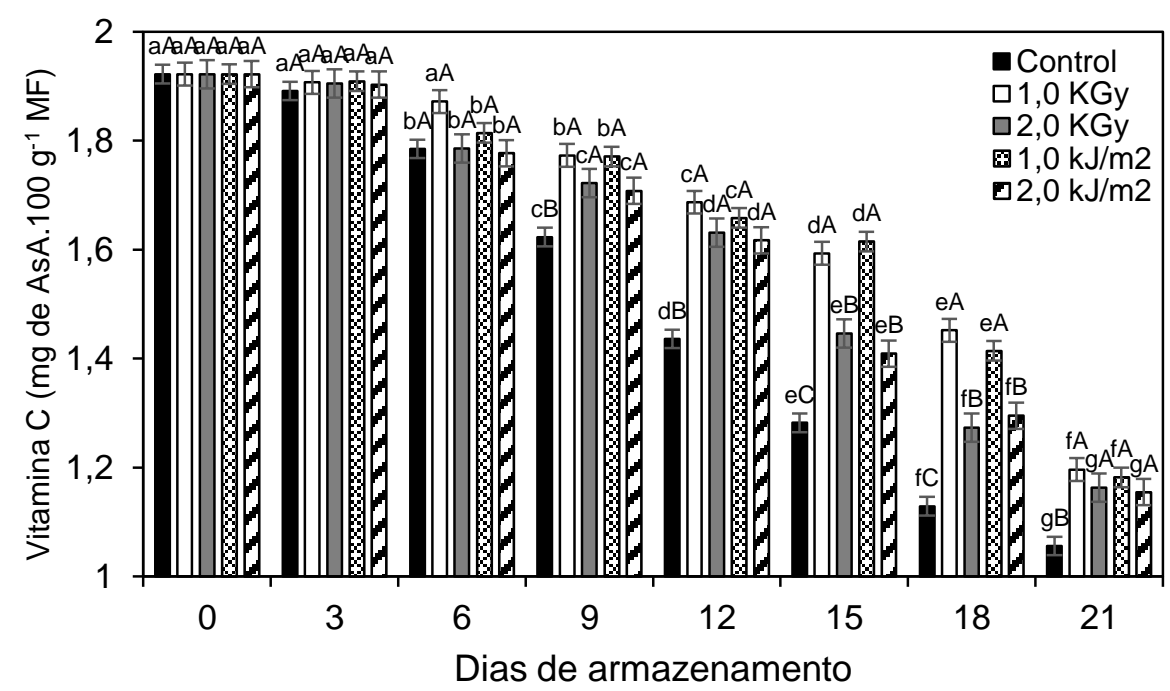


O camu-camu é um fruto rico em fonte de vitamina $\mathrm{C}$ e tem níveis muito altos de ácido ascórbico variando de 960 a $7.000 \mathrm{mg}$ de AsA.100 $\mathrm{g}^{-1} \mathrm{MF}$ (ALBERTINO et al., 2009; CHAGAS et al., 2015; GRIGIO et al., 2016).

Neste trabalho, o maior conteúdo de vitamina $C$ foi identificado no dia zero, com valor médio de $1.922 \mathrm{mg}$ de AsA.100 g ${ }^{-1} \mathrm{MF}$, valor este superior aos verificados por Rufino et al. (2010) e Pinto et al. (2013) que encontraram valores de 1.882 e $1.207 \mathrm{mg}$ de AsA.100 g $\mathrm{g}^{-1} \mathrm{MF}$ em camucamu colhidos no estádio 4 de maturação fisiológica. Já Grigio et al. (2015) relataram valores médios de $5.000 \mathrm{mg}$ de AsA.100 g ${ }^{-1} \mathrm{MF}$ em camu-camu colhidos no estado de Roraima. Esta variabilidade no teor de vitamina $\mathrm{C}$ em frutos de camu-camu é principalmente devido à região de cultivo, clima, época de colheita.

Em relação ao tempo de armazenamento, a maior decréscimo é observado nos frutos do tratamento controle, especialmente após o sexto dia, diferindo significativamente $(p<0,05)$ quando comparado aos frutos tratamentos com diferentes doses e fontes de radiação. Apesar dos frutos irradiados nas doses de 1,0 kGy e $1,0 \mathrm{~kJ} / \mathrm{m}^{2}$ apresentarem menor decréscimo no conteúdo de vitamina $C$ durante o armazenamento, estas não diferiram das demais $\left(2,0 \mathrm{kGy}\right.$ e $\left.2,0 \mathrm{~kJ} / \mathrm{m}^{2}\right)$ no último dia de análise.

Em caquis armazenados a $0{ }^{\circ} \mathrm{C}$ por 35 dias, também foi observado redução no conteúdo de vitamina $C$, no entanto, em menor quantidade quando os frutos foram submetidos a dose de 1,2 kGy de radiação gama (VIEITES et al., 2012). De modo similar, Suiubonet al. (2017) verificaram que em longans tratados com radiação UV-C $(1,0$; 2,0 e $3,0 \mathrm{~kJ} / \mathrm{m}^{2}$ ) e armazenados a $10 \stackrel{\circ}{\circ}$ por 8 dias, favoreceu menor decréscimo no conteúdo de vitamina $C$ em relação ao controle.

Com relação ao teor de antocianinas, obtiveram-se respostas diferentes quanto à dose e a fonte de radiação utilizada (Figura 7). De modo geral, o tratamento com radiação gama $(1,0 \mathrm{kGy})$ e ultravioleta $\mathrm{C}\left(2,0 \mathrm{~kJ} / \mathrm{m}^{2}\right)$ estimularam a síntese desse composto durante seis dias de armazenamento.

Figura 7.Antocianinas ( $\mathrm{mg}$, cianidina-3-glucosideo $\mathrm{g}^{-1}$ ) em camu-camu tratados com diferentes doses e fontes de radiação e armazenados sob refrigeração $10 \pm 2^{\circ} \mathrm{C}$ e $85 \pm 5 \%$ U.R durante 21 dias. Os meios seguidos pelas mesmas letras minúsculas (dias de avaliação) e maiúsculas (tratamentos), não diferem entre si pelo teste de Tukey no nível de probabilidade de $5 \%$.

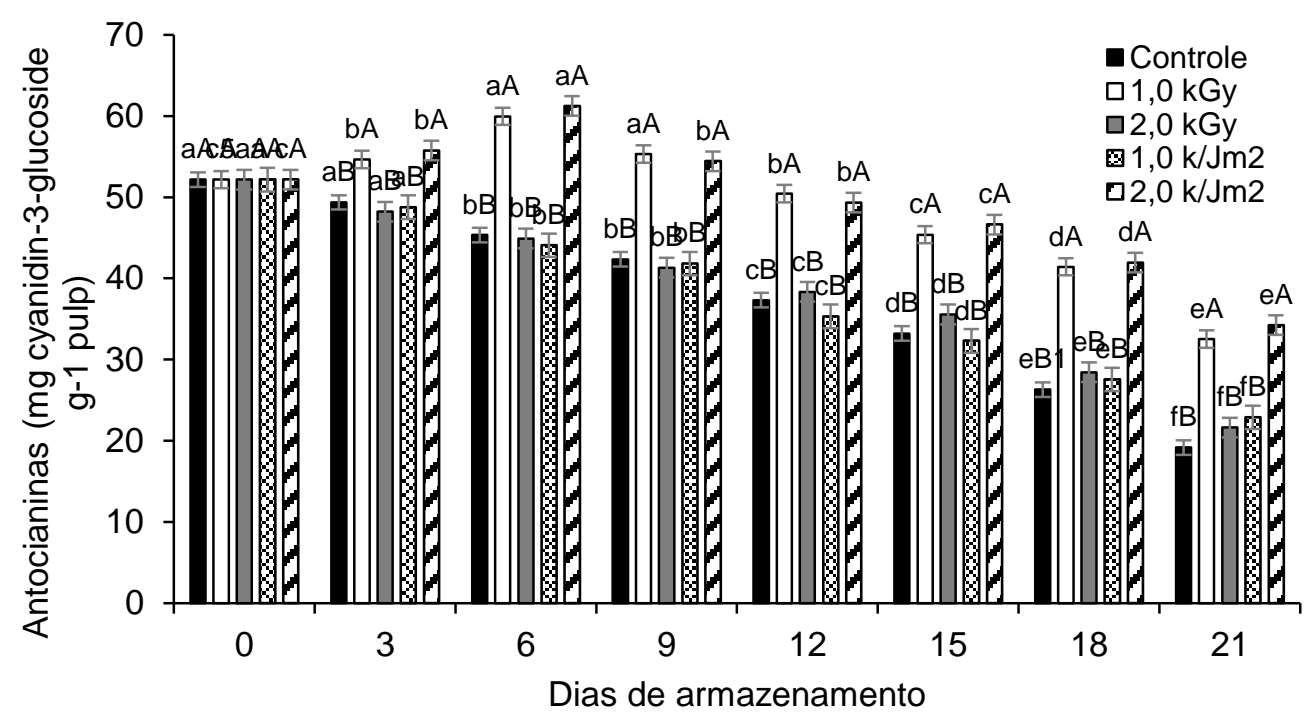

O estímulo gerado pelas dosagens de 1,0 $\mathrm{kGy}$ e $2,0 \mathrm{~kJ} / \mathrm{m}^{2}$ proporcionaram um incremento no conteúdo de antocianinas com valor de 59, 95 e 61, $\mathrm{mg}$, cianidina-3-glucosideo $\mathrm{g}^{-1}$, respectivamente, ao sexto dia de análise, além disso, observa-se menor degradação desse composto quando comparados aos demais tratamentos, nos quais houve redução ao longo de todo o período de armazenamento.
Nas plantas, as antocianinas estão relacionadas à proteção celular contra processos oxidativos causados por EROs (PEREIRA; CARDOSO, 2012). Para a saúde humana, estes têm um ótimo potencial terapêutico, são antioxidantes que ajudam na prevenção de numerosas doenças (PATRAS et al., 2010).

Neste contexto, o estímulo à produção deste fitoquímico através do estresse gerado pela 
exposição dos frutos à dose de $1,0 \mathrm{KGy}$ de radiação gama e $2,0 \mathrm{~kJ} / \mathrm{m}^{2}$ de radiação UV-C é uma indicação para a exploração dessas técnicas, visando à obtenção de frutos com melhor qualidade nutricional. No entanto, propõem-se estudos adicionais para compreender como o estresse irradiante estimula 0 metabolismo antioxidante em frutos de camu-camu.

\section{CONCLUSÕES}

O tratamento pós-colheita do camu-camu com radiação gama e ultravioleta $C$ (UV-C), especialmente nas doses de $1,0 \mathrm{kGy}$ e $1,0 \mathrm{~kJ} / \mathrm{m}^{2}$, respectivamente é eficiente em preservar a qualidade dos frutos por até 21 dias em ambiente refrigerado.

A exposição às doses de 1,0 kGy e 2,0 $\mathrm{kJ} / \mathrm{m}^{2}$ estimulou a síntese de antocianinas durante o armazenamento dos frutos.

\section{REFERÊNCIAS}

ABANTO-RODRIGUEZ, A.; PANDURO, M. P.; CHAGAS, E. A.; SAKAZAKI, R. T.; MENEZES, P. H. S. de.; ARAÚJO, W. F.; MURGA-ORRILLO. Relation between the mineral nutrients and the Vitamin $\mathrm{C}$ content in camu-camu plants (Myrciaria dubia) cultivated on high soils and flood soils of Ucayali, Peru., Scientia Agropecuaria, v. 7, n. 3, p. 297304, 2016. http://dx.doi.org/10.17268/sci.agropecu.2016.03 .18 .

ALAM KHAN, K.; ABRAHEM, M. Effect of irradiation on quality of spices.International Food Research Journal, Selangor-Malaysia, v.17, n. 1, p. 825-836, 2010.

ALBERTINO, A.; BARGE, A.; CRAVOTTO, G.; GENZINI, L.; GOBETTO, R.; VINCENTI, M. Natural origin of ascorbic acid: validation by $13 \mathrm{C}$ NMR and IRMS. Food Chemistry, v.112, p.715-720, 2009.

http://dx.doi.org/10.1016/i.foodchem.2008.05.1 $\underline{14}$.

AOAC - Association of Official Analytical Chemistry.Official methods of analysis of the Association of Official Analytical Chemistry.Washington: AOAC, 2012.

CAMPOS, A. J.; VIEITES, R. L. Conservação refrigerada de uva 'Itália' com utilização da irradiação. Revista Científica Eletrônica de Agronomia, v.17, n.1, p.31-43, 2010.
CAMPOS, A. J.; FUJITA, E.; NEVES, L. C.; VIEITES, R. L.; CHAGAS, E, A. Radiação gama e atmosfera modificada passiva na qualidade de goiabas 'Pedro Sato". Revista Brasileira deFruticultura, vol.Esp., p.350-356, 2011. http://dx.doi.org/10.1590/S010029452011000500044.

CARRILLO, M. P.; HERNÁNDEZ, M. S.; CARDONA, J. E. C.; BARRERA, J.; MARTÍNEZ, O.; FERNÁNDEZ-TRUJILLO, J. P. Prolonging postharvest quality of camu-camu (Myrciariadubia H.B.K.) as the first step in the commercial chain. Acta Horticulturae, v. 906, p. 31-36, 2011.

CHAGAS, E. A.; LOZANO, R. M. B.; CHAGAS, P. C.; BACELAR-LIMA, C. G.; GARCIA, M. I. R.; OLIVEIRA, J. V.; SOUZA, O. M.; MORAIS, B. S.; ARAÚJO, M. da.C. R. Intraspecific variability of camu-camu fruit in native populations of northern Amazonia. Crop Breeding and Applied Biotechnology, v. 15, p. 265-271, 2015.

http://dx.doi.org/10.1590/198470332015v15n4a44.

CHITARRA, M. I. F.; CHITARRA, A. B. Pós-colheita de frutos e hortaliças: fisiologia e manuseio. 2.ed. Lavras: Ed. UFLA, 2005.

CHEN, J.; WANG, X. Experimental instruction of plant physiology.Guangzhou: South Chima University of Tecnology Press,2002. p. 124.

CHEN, S.-Q.; JIANG, K. X.; CAO, B. S.; LIU, Y. Q.; CAI, F. F.; LIU, X. L.; CAO, Y.; LIU, X. Y.; SHI, W. N. Distribution of irradiated foods in China. FoodControl, v. 28, n, 2, p. 237-239, 2012. http://dx.doi.org/10.9721/KJFST.2012.44.6.673.

DAIUTO, E. R.; VIEITES, R. L.; TREMOCOLDI, M. A.; RUSSO, V. C. Taxa respiratória de abacate 'Hass' submetido a diferentes tratamentos físicos. Revistalberoamericana de TecnologíaPostcosecha, v. 10, n. 2, p. 101-109, 2010.

FAN, X.; NIEMIRA, B.A.; PRAKASH, A. Irradiation of fresh fruits and vegetables.Food Technology,v. 3, n. 2, p. 36-43, 2012.

FRANÇOSO, I. L. T.; COUTO, M. A. L.; CANNIATTIBRAZACA, S. G.; ARTHUR, V. Alterações físicoquímicas em morangos (FragariaanassaDuch.) 
irradiados e armazenados. Ciência e Tecnologia de Alimentos, v. 28, n. 3, p. 614-619, 2008. http://dx.doi.org/10.1590/S0101-

20612008000300017.

FUJITA, A.; BORGES, K.; CORREIA, R.; FRANCO, V.; GENOVESE, M. Impact of spouted beddrying on bioactive compounds, antimicrobial and antioxidant activities of commercial frozen pulp of camu-camu (MyrciariadubiaMc. Vaugh). Food Research International, v. 54, p. 495-500, 2013. https://doi.org/10.1016/j.foodres.2013.07.025

GRIGIO, M. L.; DURIGAN, M. F. B.; CHAGAS, E. A.; NASCIMENTO, C. R.; ALMEIDA, M. S. Post-harvest conservation of camu-camu fruits (Myrciariadubia(Kunth) Mc Vaugh) using different temperatures and packages. Food Science Technology, v. 35, p. 652-658, 2015. https://doi.org/10.1590/1678-457X.6788

GRIGIO, M. L.; CHAGAS, E. A.; DURIGAN, M. F. B.; SOUSA, A.A; MOTA FILHO, A. B.; CHAGAS, P. C.Determination of harvest time and quality of native camu-camu fruits (Myrciariadubia (Kunth) Mc Vaugh) during storage. Fruits, v. 71, n. 6, p. 373-378, 2016. http://dx.doi.org/10.1051/fruits/2016029.

GRIGIO, M. L.; CHAGAS, E. A.; RATHINASABAPATHI, B.; CHAGAS, P. C.; SILVA, A. R. V..; SOBRAL, S. T. M.; OLIVEIRA, R. R. Qualitative evaluation and biocompounds present in different parts of camu-camu (Myrciariadubia) fruit. African Journal of Food Science, v. 11, n. 5, p. 124-129, 2017. https://doi.org/10.5897/AJFS2016.1574

GUIMARÃES, I. C.; MENEZES, E. G. T.; ABREU, P. S.; RODRIGUES, A. C.; BORGES, P. R. S.; BATISTA, L. R.; CIRILO, M. A.; LIMA, L. C. O. Physicochemical and microbiological quality of raspberries (Rubusidaeus) treated with different doses of gamma irradiation. Food Science and Technology, v. 33, p. 316-322, 2013. http://dx.doi.org/10.1590/S0101$\underline{20612013005000040 .}$.

IMÁN, C. S.; BRAVO, Z. L.; SOTERO, S. V.; OLIVA, C. C. Contenido de vitamina $C$ en frutos de camucamu. Myrciariadubia(H.B.K) Mc Vaugh, encuatro estados de maduración, procedentes de laColección de Germoplasmadel INIA Loreto, Perú. ScientiaAgropecuaria, v. 2, n. 3, p. 123-130,
2011.

https://doi.org/10.17268/sci.agropecu.2011.03.0 1

INOUE, T.; KOMODA, H.; UCHIDA, T.; NODE, K. Tropical fruitcamu-camu (Myrciariadubia) hasanti-

oxidativeandanti-inflammatoryproperties.

JournalofCardiology, v.52, p.127-132, 2008. http://dx.doi.org/10.1016/j.jjcc.2008.06.004.

JIANG, T.; MUZAMMIL, M.; ZHENHUI, J.; XIANYING J.; YING, L. T. Influence of UV-C treatment on antioxidant capacity, antioxidant enzyme activity and texture of postharvest shiitake (Lentinusedodes) mushrooms during storage. Postharvest Biology and Thecnology, $v$. 56, n. 3, p. 209-215, 2010. https://doi.org/10.1016/j.postharvbio.2010.01.01 $\underline{1}$

KHADEMI, O.; ZAMANI, Z.; POOR AHMADI, E.; KALANTARI, S. Effect of UV-radiation on postharvest physiology of persimmon fruit (Diospyros kaki Thunb.) cv.'Karaj' during storage at cold temperature. International Food Research Journal, v. 20, p. 247-253, 2013.

MYODA, T.; FUJIMURA, S.; PARK, B.; NAGASHIMA, T.; NAKAGAWA, J. NISCHIZAWA, M. Antioxidativeandantimicrobialpotentialofresidues ofcamu-camujuiceproduction.JournalofFood,

Agriculture\&Environment, v.8, n.2, p.304-307, 2010.

NIMITKEATKAI, H.; KULTHIP, J. Effect of sequential UV-C irradiation on microbial reduction and quality of fresh-cut dragon fruit.InternationalFoodResearchJournal, v. 23, n. 4, p. 1818-1822, 2016.

OLIVEIRA, J.; SILVA, I. G.; SILVA, P. P. M.; SPOTO, M. H. F. Atmosfera modifi cada e refrigeração para conservação pós-colheita de camu-camu. Ciência Rural, Santa Maria, v.44, n.6, p.11261133, 2014. http://dx.doi.org/10.1590/S010384782014000600028.

PATARO, G.; SINIK, M.; CAPITOLI, M. M.; DONSI, G.; PATARO, G. The influence of post-harvest UV$C$ and pulsed light treatments on quality and antioxidant properties of tomato fruits during storage. InnovativeFood Science and Emerging 
Technologies, v. 30, p. 103-111, 2015. http://dx.doi.org/10.1016/i.ifset.2015.06.003.

PATRAS, A.;BRUNTON, N. P.; O'DONELL, C.; KUMAR, T. B. Effect of thermal processing on anthocyanin stability in foods; mechanisms and kinetics of degradation. Trends in Food Science \& Technol., v.21, p.3- 11, 2010. http://dx.doi.org/10.1016/j.tifs.2009.07.004.

PEREIRA, R. J.; CARDOSO, M. G. Vegetable secondary metabolites and antioxidants benefits.Journal of Biotechnology and Biodiversity, v. 3, n. 4, p. 146-152, 2012.

PINTO, P. M.; JACOMINO, A. P.; SILVA, S. R.; ANDRADE, C. A. W. Ponto de colheita e maturação de frutos de camu-camu colhidos em diferentes estádios. Pesquisa Agropecuária Brasileira, v.48, n.6, p.605-612, 2013. http://dx.doi.org/10.1590/S0100204X2013000600005.

POMBO, M. A., DOTTO, M. C., MARTINEZ, G. A., CIVELLO, P. M. UV-C irradiation delays strawberry fruit expression of genes involved in cell wall degradation. Postharvest Biology and Technology, v. 51, p. 141-148, 2009. https://doi.org/10.1016/i.postharvbio.2008.07.00 7.

RUFINO, M. S. M.;ALVES, R. E.; BRITO, E. S.; PÉREZ-JIMÉNEZ, J.; SAURA-CALIXTO, F.; MANCINIFILHO,

J.Bioactivecompoundsandantioxidantcapacities non-traditional tropical fruitsfromBrazil. Food Chemistry, v.121, n. 4, p.996-1002, 2010. https://doi.org/10.1016/j.foodchem.2010.01.037

SANCHES, A. G.; SILVA, M. B.; MOREIRA, E. G. S.; COSTA, J. M. CORDEIRO, C. A. M. Radiação uv-c na longevidade pós-colheita de tangerinas sob refrigeração. Sci. Agrar. Parana., Marechal v. 15, n. 3, p. 338-344, 2017. http://dx.doi.org/10.18188/1983-

1471/sap.v15n3p338-344.

SANCHES, A. G.; COSTA, J. M.; SILVA, M. B.; MOREIRA, E. G. S.; SANTANA, P. J. A.; CORDEIRO, C. A. M. Aspectos qualitativos e amadurecimento do araçá amarelo tratado com radiação UV-C. Nativa, v. 5, n. 5, p. 303-310, 2017. http://dx.doi.org/10.5935/2318-7670.v05n05a01.
SILVA, S. R.; BEZERRA, D. N. F.; BASSAN, M. M.; CANTUARIAS-AVILÉ, T. ARTHUIR, V. Postharvest of irradiated tahiti lime fruits.Revista Brasileira de Fruticultura, v. 38, n. 4, p. e-272, 2015. http://dx.doi.org/10.1590/0100-29452016272.

SILVA, F. A. S.; AZEVEDO, C. A. V.The Assistat Software Version 7.7 and its use in the analysis of experimental data.African Journal Agricola Research., v.11, n.39, p.3733-3740, 2016. http://dx.doi.org/10.5897/AJAR2016.11522.

SINGH, A.; SINGH, D.; SINGH, R. Shelf life extension of tomatoes by gamma radiation.Radiation Science and Technology, v. 2, n. 2, p. 17-24, 2016. http://dx.doi.org/10.11648/j.rst.20160202.12.

STEFANOVA, R.; VASILEV, N. V.; SPASSOV, S. L. Irradiation of food, current legislation framework, and detection of irradiated foods.Food Analytical Methods, v. 3, n. 2, p. 225-236, 2010. https://doi.org/10.1007/s12161-009-9118-8.

SUIUBON, S.; SUPAPVANICH, S.; PROMYOU, S. Postharvest quality maintenance of longan fruit by ultra violet-C incorporated with salicylic acid application. Emirates Journal of Food and Agriculture, v. 29, n. 3, p. 179-187, 2017. http://dx.doi.org/10.9755/ejfa.2016-09-1269.

TEIXEIRA, L. N.; STRINGHETA, P. C.; OLIVEIRA, F. A. Comparação de métodos para quantificação de antocianinas. Revista Ceres, v. 55, n. 4, p. 297304, 2008.

TEZOTTO-ULIANA, J. V.; SILVA, P. P. M.; KLUGE, R. A.; SPOTO, M. H. F. Radiação Gama em Produtos de Origem Vegetal.Revista Virtual de Quimica, v. 7 n. 1, p. 267-277, 2015. http://dx.doi.org/10.5935/1984-6835.20150013.

VIEITES, R. L.; PICANÇO, N. F. M.; DAIUTO, E. R. Radiação gama na conservação de caqui 'giombo', destanizado e frigoarmazenado. Rev. Bras. Frutic., v. 34, n. 3, p. 719-726, 2012. http://dx.doi.org/10.1590/S0100$\underline{29452012000300010 .}$.

YADAV, M. K.; PATEL, N. L.; PATEL, D. P.; PARMAR, M. R. Alphonso mango conservation through exposure to gamma radiation. African Journal of Food Science, v. 9, n. 3, p. 97-102, 2015. http://dx.doi.org/10.5897/AJFS2014.1245. 
YUYAMA, K.; YAYAMA, L. K. O.; VALENTE, J. P.; SILVA, A. C.; AGUIAR, J. P. L.; FLORES, W. B. C.; LIMA, C. G. B. C. Camu-camu. Jaboticabal: FUNEP, 2010. 50 p.

ZANATTA, C. F.; MERCADANTE, A. Z. CarotenoidcompositionfromtheBrazilian tropical fruitcamu-camu (Myrciariadubia). Food Chemistry, v.101, n. 1, p.1526-1532, 2007. https://doi.org/10.1016/j.foodchem.2006.03.043

Recebido para publicação em 11/08/2017

Revisado em 22/03/2018

Aceito em 26/06/2018 